\title{
A COMPARISON OF THE INTRANIGRAL DISTRIBUTION OF NIGROTECTAL NEURONS LABELED WITH HORSERADISH PEROXIDASE IN THE MONKEY, CAT, AND RAT ${ }^{1}$
}

\author{
ROBERT M. BECKSTEAD, STEPHEN B. EDWARDS, AND ANTHONY FRANKFURTER
}

Departments of Anatomy and Neurosurgery, University of Virginia School of Medicine, Charlottesville, Virginia 22908

\begin{abstract}
The location of neurons in the substantia nigra's pars reticulata (SNR) that send their axons to the superior colliculus was compared in the monkey, cat, and rat using the horseradish peroxidase (HRP) retrograde cell-labeling method. Although several cases of large, unilateral HRP deposits in the superior colliculus show that in all three species, the nigrotectal cells are confined, for the most part, to the rostral one-half of SNR, the following differences were noted in the precise location of the nigrotectal neurons and in the degree of bilaterality of the nigrotectal projection. In the monkey, labeled nigrotectal cells were particularly numerous in the extreme rostrolateral portion of SNR. From this region of high concentration, a progressively decreasing number of cells spreads medially in a ventral stratum immediately dorsal to the pes pedunculi. No labeled cells were found in the extreme medial part of SNR. A substantial number of HRP-positive cells were present in the contralateral SNR in a similar distribution. In the cat, labeled cells were less selectively localized in SNR's mediolateral expanse, being distributed more or less randomly in its middle portion with a scattering of cells in both medial and lateral parts of SNR. Although some cell labeling occurred in the contralateral SNR, it was less substantial than in the monkey. In the rat, the HRPpositive cells were especially concentrated throughout the mediolateral extent of a ventral stratum of SNR immediately dorsal to the pes pedunculi. Although some cells were located more dorsally, they were far fewer in number and consistently less heavily labeled. Only one or two labeled cells could be detected in the contralateral SNR of the rat. These anatomical differences suggest that the influence of the corpus striatum on the tectal control of orienting responses may vary considerably from one mammalian species to the next.
\end{abstract}

Contrary to earlier conclusions (Afifi and Kaelber, 1965; Carpenter and Strominger, 1967; Carpenter and Peter, 1972; Carpenter et al., 1976), recent anatomical evidence has documented the existence of a prominent, intramesencephalic projection from the pars reticulata of the substantia nigra to the intermediate, and possibly deep, layers of the superior colliculus in the rat (Faull and Mehler, 1978; Bentivoglio et al., 1979; Beckstead et al., 1979), cat (Graybiel and Sciascia, 1975; Hopkins and Niessen, 1976; Rinvik et al., 1976; Edwards et al., 1979), and monkey (Jayaraman et al., 1977). Remarkably, in these many studies, there appears to be little consistency in the reported location of nigrotectal cells within the pars reticulata. This lack of agreement could be due to technical differences from one study to the next or could reflect a significant topological difference across species. In an attempt to assess the latter possibility, we charted the intranigral location of retrogradely labeled neurons after large, unilateral, horseradish peroxidase (HRP) deposits were made in the superior colliculi of rats, cats,

' This work was supported by National Institutes of Health Grants 5 S07 KR05431-18 and NS 11254 . and monkeys. We here report not only interspecies differences in the location of nigrotectal cells but, further, an increasing degree of bilaterality of the projection from rodent to primate.

\section{Materials and Methods}

This report is based on observations made in three monkeys (two cynomolgus, one squirrel), four cats, and eight hooded rats which are part of a larger series of experiments designed to examine the organization of various tectal inputs. The present cases were selected because the HRP (Boehringer or Sigma type IX) deposit in each involved nearly all of the superior colliculus on one side of the brain stem with only minimal or no apparent encroachment on adjacent cell groups. The animals had survived 36 to $48 \mathrm{hr}$ before transcardial perfusion with $0.1 \mathrm{M}$ phosphate buffer $(\mathrm{pH} 7.4$ ) containing $1 \%$ paraformaldehyde and $1.5 \%$ glutaraldehyde followed by phosphate buffer containing only $10 \%$ sucrose. The brains were removed and cut into transverse sections of $40 \mu \mathrm{m}$ thickness on the freezing microtome. The sections were incubated with tetramethylbenzidine according to the method of Mesulam (1978) or with O-dianisidine 
according to the method of de Olmos (1977), mounted on gelatin-coated slides, and counterstained with either neutral red or cresyl echt violet using acetone rather than alcohol to prevent fading of the HRP reaction product. Serial sections through the midbrain were examined in each case and the labeled neurons were charted on projection drawings.

\section{Results}

The present cases were chosen for description because, in each, the HRP deposit involved all layers of the superior colliculus on one side of the brain stem and did not appear to involve adjacent cell groups such as the pretectal nuclei or the inferior colliculus. Although, in some cases, the HRP deposit involves also the dorsolateral quadrant of the central gray substance, the pattern of nigral cell labeling is essentially the same as when the central gray substance is not involved. A typical HRP deposit in the monkey's superior colliculus is shown in Figure 1 . Since the pattern of intranigral cell labeling is the same within a given species, the cases for each species will be described together.

Monkey. Labeled cells in the ipsilateral substantia nigra of the monkey are located only in the pars reticulata

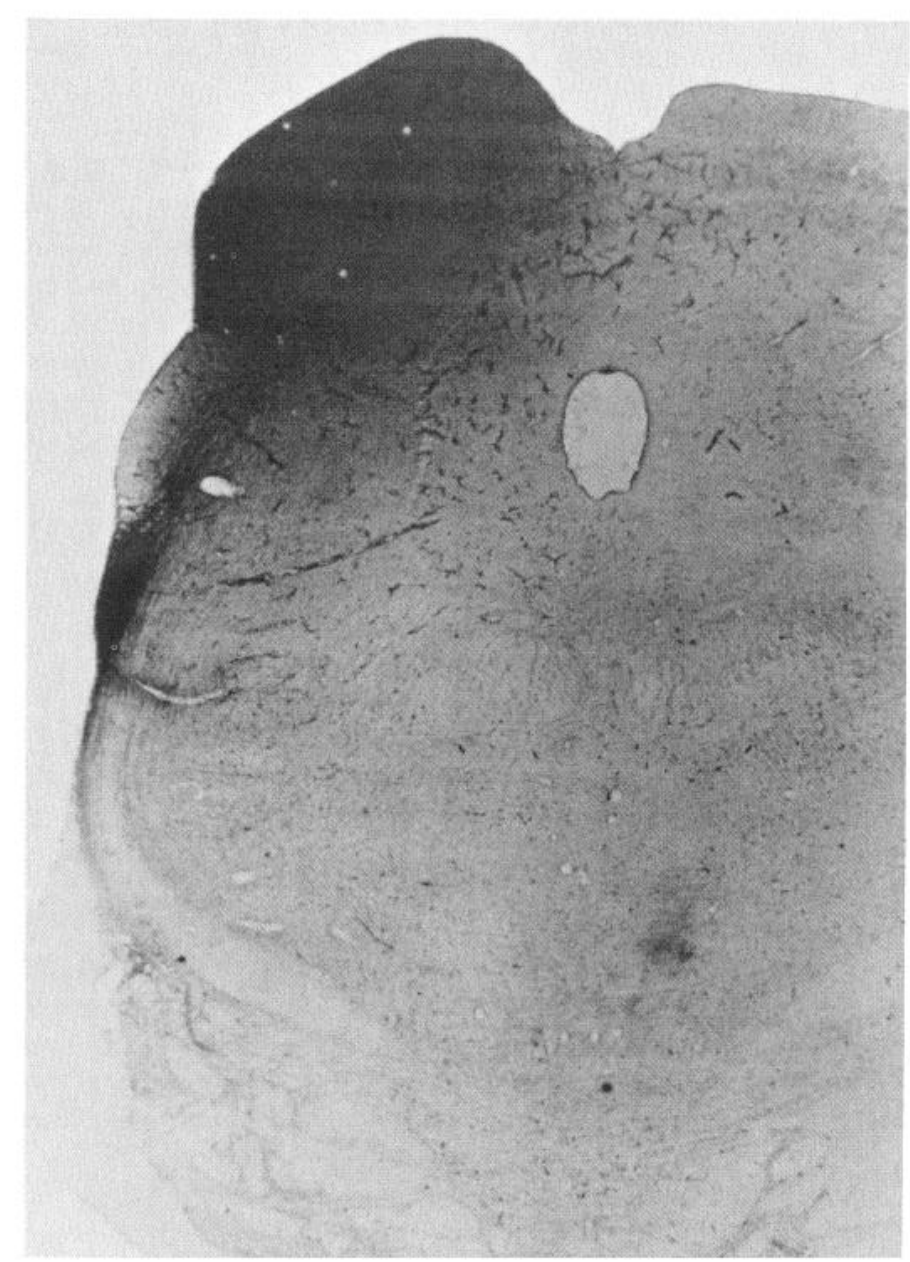

Figure 1. A light field photomicrograph showing the deposition of HRP-tetramethylbenzidine reaction product in the monkey's superior colliculus.
(SNR) where they are distributed from its rostral pole caudalward to approximately a frontal plane at which the caudalmost roots of the oculomotor nerve exit the brain stem in the interpeduncular fossa (Fig. $2 A$ ). Near its rostral pole, several HRP-positive cells are scattered throughout SNR, but at progressively more caudal levels, the labeled cells congregate in the extreme lateral margin of SNR. From this lateral region of high concentration of nigrotectal cells, a diminishing number of labeled cells extends medially about halfway across the mediolateral expanse of SNR in a ventral stratum immediately supraadjacent to the pes pedunculi. No cell labeling is present in more medial or dorsal territories of SNR nor are any neurons labeled in the pars compacta of the substantia nigra.

The labeled SNR neurons are generally of irregular shape and medium size (20 to $30 \mu \mathrm{m}$ somal diameter) with three or four major dendrites, and they exhibit a tendency to cluster into aggregates of five to 10 cells (Fig. $3 A$ ). In total, approximately 750 to 800 labeled neurons are present in the ipsilateral SNR in any one of the three monkey cases.

On the side contralateral to the collicular HRP deposit, labeled neurons are located only in the rostrolateral corner of SNR-an area corresponding to the region of densest ipsilateral nigral labeling (Fig. $3 B$ ). The number of labeled cells per case in the contralateral SNR ranges from about 120 to 150 .

Cat. In the cat, as is the case for the monkey, all of the retrogradely labeled nigral cells are confined to the rostral one-third to one-half of the ipsilateral SNR (Fig. $2 B$ ). However, rather than being concentrated in the lateralmost zone of SNR, the labeled neurons are instead scattered throughout the mediolateral and dorsoventral expanse of SNR, with a slightly higher density ventrally near the middle of the mediolateral expanse. As in the monkey, no cell labeling occurred in the substantia nigra's pars compacta. The total number of HRP-positive cells in the cat's ipsilateral SNR is on the order of 950 to 1200 . Only a small number ( 80 to 100 ) of labeled neurons could be found in the contralateral SNR. The morphology of the HRP-filled cells is similar, in general, to that described for the monkey (Fig. $4 A$ ).

Rat. Again, like the monkey and cat, retrogradely labeled cells in the rat's ipsilateral SNR are confined, for the most part, to its rostral one-half. The pattern of cell labeling, however, is strikingly different such that the zone of labeled cells consists of a thin stratum immediately dorsal to the pes pedunculi that extends across the entire mediolateral expanse of SNR (Fig. $2 C$ ). At the rostromedial and caudolateral edges of this layer of labeled cells are small clumps of HRP-positive neurons. Very few labeled cells are present in the larger, more dorsal regions of SNR, and these cells are consistently more faintly labeled with the HRP reaction product. The total number of labeled cells in the ipsilateral SNR ranges from about 260 to 300 . No cell labeling is detectable in the pars compacta and, with the exception of one case in which two labeled cells are present, no neurons are labeled in the contralateral SNR. The HRP-positive cells in the rat's SNR are smaller in absolute terms (15 to 20 $\mu \mathrm{m}$ somal diameter) than those in the monkey or cat and 


\section{MONKEY}

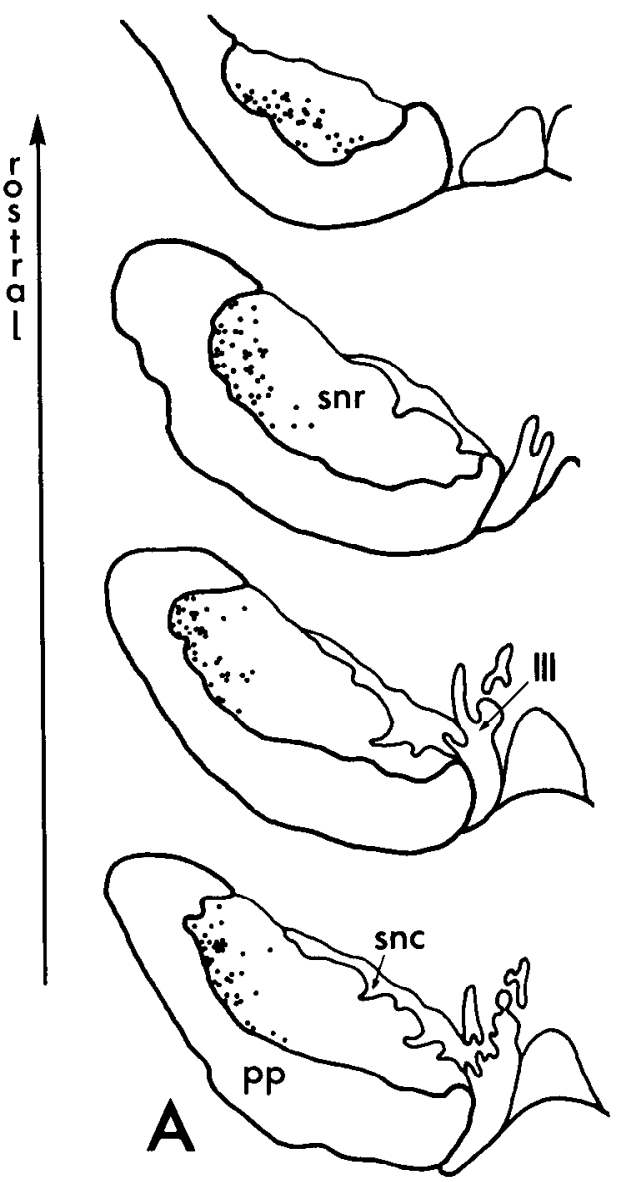

CAT
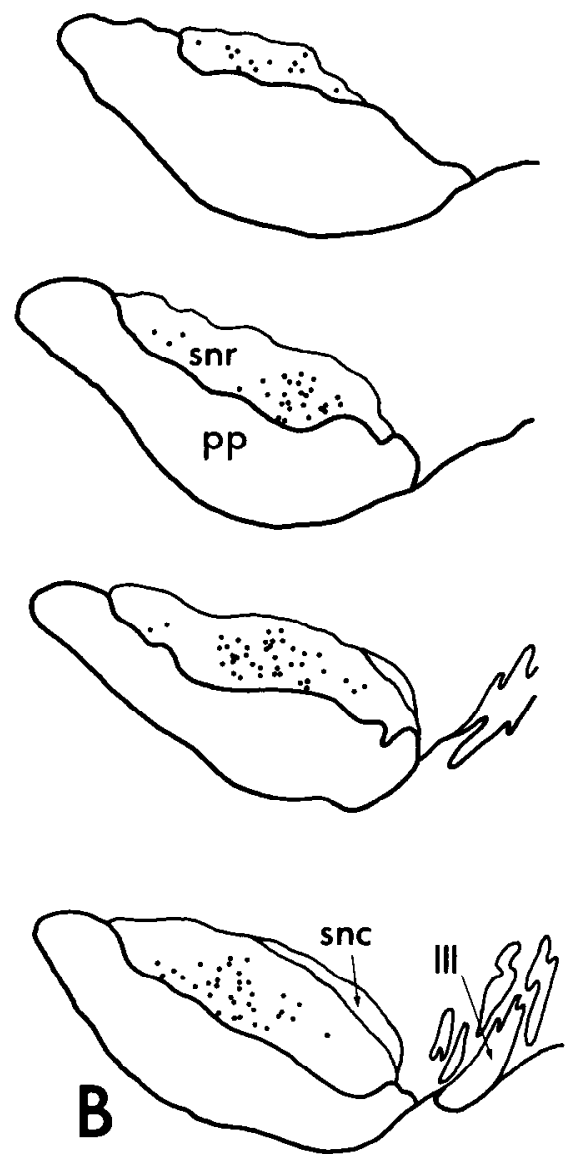

RAT
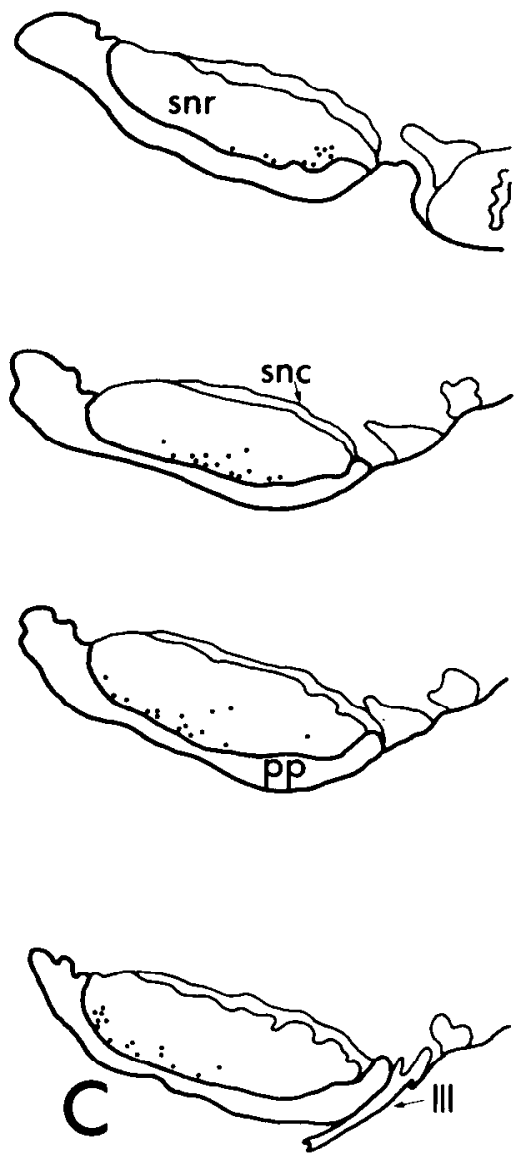

Figure $2 .^{2}$ Chartings of selected transverse sections through the substantia nigra showing the distribution of HRP-positive cells $(d o t s)$ in representative cases from $(A)$ a monkey, $(B)$ a cat, and $(C)$ a rat. The nigras from the three species are not drawn to scale.

appear to have only two or three major dendritic processes, which are usually oriented parallel to the surface of the pes pedunculi (Fig. $4 B$ ).

\section{Discussion}

The present results demonstrate that, in all three of these common laboratory mammals, the nigrotectal neurons are restricted to the rostral half of SNR, and in the rat and monkey, they are confined to an even more circumscribed zone of SNR. Our observation that the rat's nigrotectal cells are located ventrally and only on the ipsilateral side agrees with that of Faull and Mehler (1978), and our observations for the cat confirm those of Rinvik et al. (1976) and Edwards et al. (1979). Our HRP data also confirm the conclusions of éarlier anterograde autoradiographic findings that the nigrotectal projection is ipsilateral in the rat (Beckstead et al., 1979) and substantially bilateral in the monkey (Jayaraman et al., 1977).

The present finding of spatial segregation of nigrotectal

\footnotetext{
${ }^{2}$ The abbreviations used on the figures are: pp, pes pedunculi; snc, pars compacta of the substantia nigra; snr, pars reticulata of the substantia nigra; III, oculomotor nerve.
}

neurons in the rat and especially the monkey suggests that many if not most (perhaps all in the monkey) of the tectal-projecting neurons constitute a nigral cell population that is distinct from the remaining, larger population of SNR cells that send axons to either the thalamus, the pedunculopontine nucleus, or both. Double retrograde labeling experiments using fluorescent dyes in rats (Bentivoglio et al., 1979) and electrophysiological experiments in cats (Anderson and Yoshida, 1977) have suggested that a substantial number of SNR cells that send axons to the superior colliculus also send an axon collateral to the thalamus. No such data have appeared as yet for the monkey. It is noteworthy that, in the double label experients of Bentivoglio et al. (1979), the neurons labeled only with the tectally injected dye lie ventrally in the rostral part of SNR, and those marked only with thalamically injected dye lie dorsally throughout SNR, with the smaller number of doubly labeled cells located at middle depths of SNR.

There are scant data presently available on the degree of collateralization of nigral axons with regard to other targets of SNR cells such as the pedunculopontine nucleus. However, the HRP data of Hopkins and Niessen (1976) for the rat, cat, and monkey and that of Rinvik et 

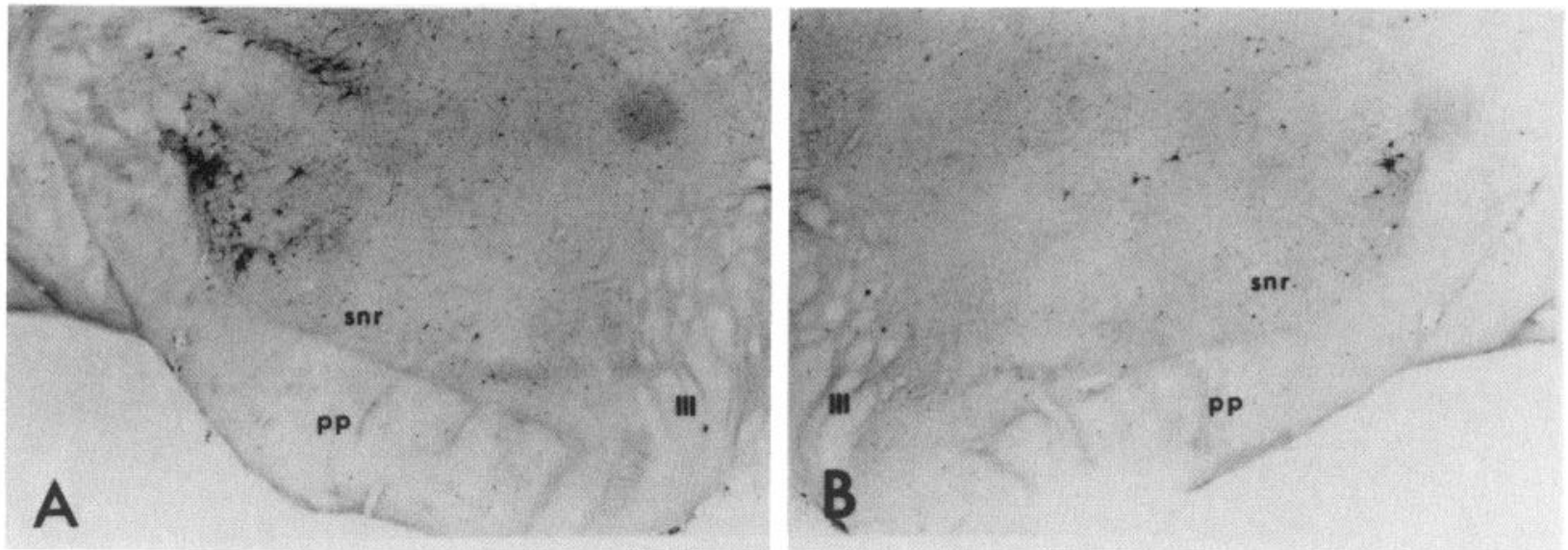

Figure 3. Light field photomicrographs of HRP-positive neurons in the monkey's $(A)$ ipsilateral and $(B)$ contralateral SNR. Magnification $\times 25$.
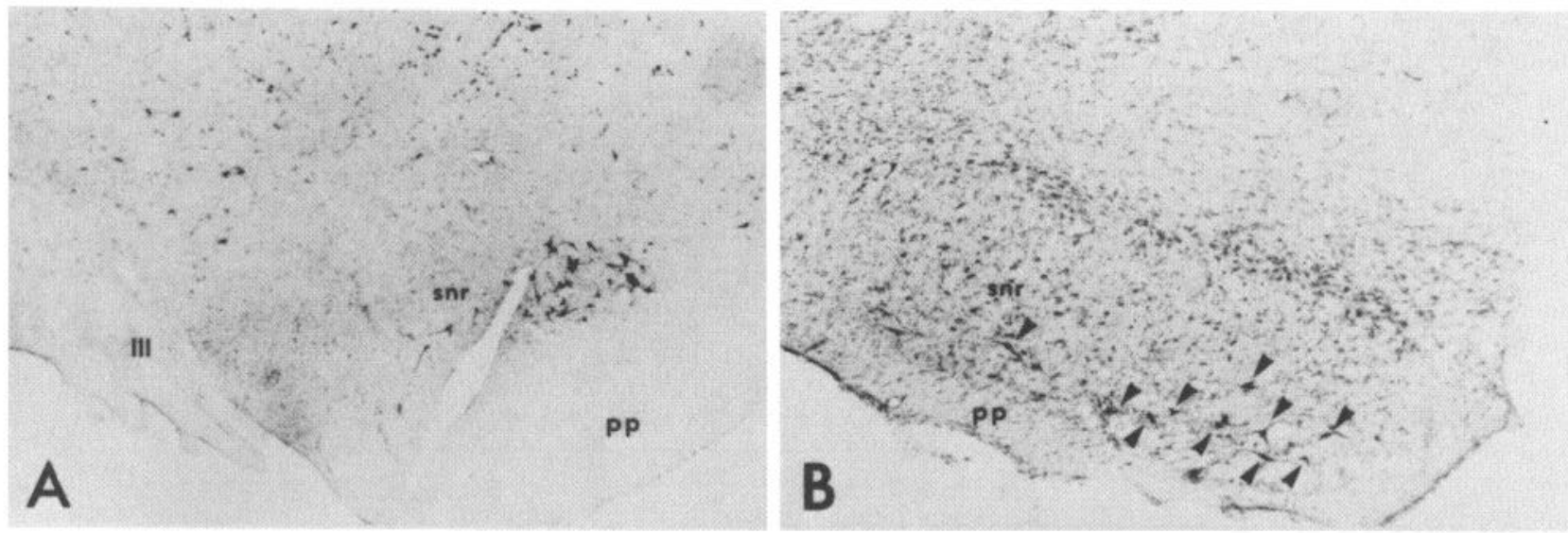

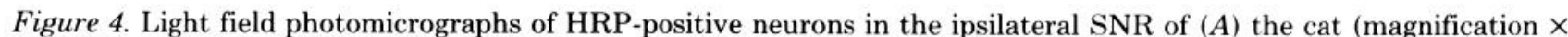
and $(B)$ the rat (magnification $\times 50$ ).

al. (1976) for the cat suggest that the nigral neurons that project to more caudal levels of the brain stem are located in caudal regions of SNR rather than in the rostral part which contains the nigrotectal cells.

Regardless of whether nigrotectal cells also send an axon collateral to the thalamus or elsewhere, it is clear from the present study that the nigrotectal neurons in all three species are a spatially distinct subset since they are confined to the rostral half of SNR, whereas nigrothalamic cells are present throughout the rostrocaudal extent of SNR (Faull and Mehler, 1978; Herkenham, 1979; Bentivoglio et al., 1979; Rinvik, 1975; Carpenter et al., 1976; Carpenter and Peter, 1972).

Anterograde studies by the autoradiographic method have shown that, in all three species, the nigrotectal axons terminate in the intermediate and possibly the deep layers of the tectum, distributing in an alternately high and low density, "puffs and holes" pattern (Graybiel and Sciascia, 1975; Jayaraman et al., 1977; Beckstead et al., 1979). These same studies showed that the terminal distribution of nigrotectal axons is somewhat more pronounced in the lateral part of the superior colliculus. It may be salient to note that most of the tectospinal neurons, i.e., the tectal cells that, in all likelihood, influ- ence the motor components of orienting head movement (Anderson et al., 1971, 1972; Abrahams and Rose, 1975), are located in the lateral part of the intermediate and deep tectal layers (Castiglioni et al., 1978; Rhoades and DellaCruce, 1980; Edwards et al., 1980). Indeed, York and Faber (1977) have suggested that the nigrotectal projection might function in relation to visual orienting behavior

It has been demonstrated that the nigrotectal neurons synthesize $\gamma$-aminobutyric acid (Vincent et al., 1978) and are, therefore, probably inhibitory. This observation is compatible with the present finding that none of the labeled nigrotectal cells are located among the dopaminergic neurons of the substantia nigra's pars compacta. These findings should not be taken to imply that there is no dopamine influence on the tectum, however, because the dopaminergic pars compacta neurons project heavily to the striatum, which, in turn, is almost certainly a major source of afferent axons to the nigrotectal cells in SNR. Hence, the motor dysfunctions such as turning behavior that are observed in cases of dopamine depletion may express themselves partially by way of tectal output neurons.

The topological restriction of nigrotectal neurons 
within SNR raises the possibility that they receive afferent information that is, in some ways, different from that impinging upon the other neurons of SNR. This seems especially likely to be true for striatonigral afferent axons which are known to distribute in SNR in a topographically organized manner (Domesick, 1977; Szabo, 1962). For example, Domesick (1977) has shown that, in the rat, the ventralmost stratum of SNR, where the nigrotectal cells predominate, is projected upon by dorsal regions of the nucleus caudatoputamen, whereas progressively more dorsal regions of SNR, where nigrothalamic cells predominate, receive striatal afferents from progressively more ventral regions of the caudatoputamen. The differences in the precise intranigral location of nigrotectal cells and in the bilaterality of the nigrotectal projection among the three species raises the further possibility of interspecies differences in the function of the nigrotectal projection. It is of paramount importance to determine whether the striatal input to the nigrotectal cells in each species arises from functionally homologous cell groups in the striatum. For instance, do the striatal cells whose axons contact the nigrotectal cells receive their cortical input from functionally homologous cortical areas?

The range of motor strategies for orientation behavior and the relative efficacy of a stimulus in a particular modality to trigger an orienting response are sufficiently different from one mammalian species to the next that it would not be unrealistic to expect qualitative differences in the character of information reaching and being conveyed by nigrotectal neurons in each species. Whether such interspecies functional differences are present in the nigrotectal projection system requires further pairstaking investigation. In this context, the present work provides a point of departure by delimiting the territory of nigrotectal neurons in SNR for three common laboratory species.

\section{References}

Abrahams, V. C., and P. K. Rose (1975) Projections of extraocular, neck muscle, and retinal afferents to superior colliculus in the cat: Their connection to cells of origin of tectospinal tract. J. Neurophysiol. 38: 10-18.

Afifi, A. K., and W. W. Kaelber (1965) Efferent connections of the substantia nigra in the cat. Exp. Neurol. 11: 474-482.

Anderson, M., and M. Yoshida (1977) Electrophysiological evidence for branching nigral projections to the thalamus and the superior colliculus. Brain Res. 137: 361-364.

Anderson, M. E., M. Yoshida, and V. J. Wilson (1971) Influence of superior colliculus on cat neck motoneurons. J. Neurophysiol. 34: 898-907.

Anderson, M. E., M. Yoshida, and V. J. Wilson (1972) Tectal and tegmental influences on cat forelimb and hindlimb motoneurons. J. Neurophysiol. 35: 462-470.

Beckstead, R. M., V. B. Domesick, and W. J. H. Nauta (1979) Efferent connections of the substantia nigra and ventral tegmental area in the rat. Brain Res. 175: 191-217.

Bentivoglio, M., D. Van Der Kooy, and H. G. J. M. Kuypers (1979) The organization of the efferent projections of the substantia nigra in the rat. A retrograde fluorescent double labeling study. Brain Res. 130: 383-386.
Carpenter, M. B., and P. Peter (1972) Nigrostriatal and nigrothalamic fibers in the rhesus monkey. J. Comp. Neurol. 144: 93-116.

Carpenter, M. B., and N. L. Strominger (1967) Efferent fiber projections of the subthalamic nucleus in the rhesus monkey. A comparison of the efferent projections of the subthalamic nucleus, substantia nigra and globus pallidus. Am. J. Anat. 121: 41-72.

Carpenter, M. B., K. Nakano, and R. Kim (1976) Nigrothalamic projections in the monkey demonstrated by autoradiographic technics. J. Comp. Neurol. 165: 401-416.

Castiglioni, A. J., M. C. Gallaway, and J. D. Coulter (1978) Spinal projections from the midbrain in monkey. J. Comp. Neurol. 178: 329-346.

de Olmos, J. S. (1977) An improved HRP method for the study of central nervous connections. Exp. Brain Res. 29: 541-551.

Domesick, V. V. (1977) The topographic organization of the striatonigral connections in the rat. Anat. Rec. 187: 567.

Edwards, S. B., C. L. Ginsburgh, C. K. Henkel, and B. E. Stein (1979) Sources of subcortical projections to the superior colliculus in the cat. J. Comp. Neurol. 184: 309-330.

Edwards, S. B., C. K. Henkel, and K. S. Kersey (1980) Structural characteristics of superior colliculus neurons governing head and pinna movements in the cat. Neurosci. Lett. (Suppl.) 5: 337.

Faull, R. L. M., and W. R. Mehler (1978) The cells of origin of nigrotectal, nigrothalamic and nigrostriatal projections in the rat. Neuroscience 3: 989-1002.

Graybiel, A. M., and T. R. Sciascia (1975) Origin and distribution of nigrotectal fibers in the cat. Soc. Neurosci. Abstr. 1: 174.

Herkenham, M. (1979) The afferent and efferent connections of the ventromedial thalamic nucleus in the rat. J. Comp. Neurol. 183: 487-518.

Hopkins, D. A., and L. W. Niessen (1976) Substantia nigra projections to the reticular formation, superior colliculus and central gray in the rat, cat and monkey. Neurosci. Lett. 2: 253-259.

Jayaraman, A., R. R. Batton, and M. B. Carpenter (1977) Nigrotectal projections in the monkey: An autoradiographic study. Brain Res. 135: 147-152.

Mesulam, M.- M. (1978) Tetramethyl benzidine for horseradish peroxidase neurohistochemistry: A non-carcinogenic blue reaction-product with superior sensitivity for visualizing renal afferents and efferents. J. Histochem. Cytochem. 26: 106-117.

Rhoades, R. W., and D. R. DellaCruce (1980) Cells of origin of the tectospinal tract in the golden hamster: An anatomical and electrophysiological investigation. Exp. Neurol. 67: 163180.

Rinvik, E. (1975) Demonstration of nigrothalamic connections in the cat by retrograde axonal transport or horseradish peroxidase. Brain Res. 90: 313-318.

Rinvik, E., I. Grofová, and O. P. Ottersen (1976) Demonstration of nigrotectal and nigrorecticular projections in the cat by axonal transport of proteins. Brain Res. 112: 388-394.

Szabo, J. (1962) Topical distribution of the striatal afferents in the monkey. Exp. Neurol. 5: 21-36.

Vincent, S. R., T. Hattori, and E. G. McGeer (1978) The nigrotectal projection: A biochemical and ultrastructural characterization. Brain Res. 151: 159-164.

York, D. H., and J. E. Faber (1977) An electrophysiological study of nigro-tectal relationships: A possible role in turning behavior. Brain Res. 130: 383-386. 